\title{
DETERMINATION OF THE ABSOLUTE CONFIGURATION OF MONO- SACCHARIDES IN COMPLEX CARBOHYDRATES BY CAPILLARY G.L.C.
}

Gerrit J. Gerwig, Johannis P. Kamering, and Johannes F. G. Vliegenthart

Laboratory of Organic Chemistry, University of Utrecht (The Netherlands)

(Received February 26th, 1979; accepted for publication, March 26th, 1979)

\section{ABSTRACT}

The absolute configuration of neutral monosacchazides, 2-acetamido-2-deoxy sugars, and uronic acids can be determined by capillary g.l.c. on SE-30 after glycosidation with (-)-2-butanol and protection of the remaining polar groups. The method is illustrated by application to mixtures of the constituent sugars of the capsular polysaccharide from Klebsiella type 57, $\alpha_{1}$-acid glycoprotein, mucus glycoproteins, and the methylated, acidic polysaccharide from the coccoliths of Emiliania huxleyi (Lohmann) Kamptner.

\section{INTRODUCTION}

Determination of the absolute configuration of neutral monosaccharides can be achieved ${ }^{1}$ by their conversion into the trimethylsilylated (-)-2-butyl $\alpha \beta$-glycosides followed by capillary g.l.c. on SE-30; $D$ and $L$ monosaccharide derivatives give rise to different multiple-peak patterns. A similar approach using acetylated $(+)-2$-octyl glycosides has been described ${ }^{2}$.

We now report an extension of our method to 2-acetamido-2-deoxy sugars and uronic acids.

\section{RESULTS AND DISCUSSION}

Figs. 1-5 show the g.l.c. patterns of the trimethylsilylated (-)- and (+)-2butyl glycosides of 2-acetamido-2-deoxy-D-mannose, 2-acetamido-2-deoxy-D-galactose, 2-acetamido-2-deoxy-D-glucose, D-galacturonic acid (as the 2-butyl ester), and D-glucuronic acid (as the 2-butyl ester and lactone), respectively, on SE-30 (135 $\rightarrow$ $220^{\circ}$ at $1 \% \mathrm{~min}$ ). As mentioned earlier ${ }^{1}$, on non-chiral stationary phases, (-)-alkyl D-glycosides and $(+)$-alkyl L-glycosides are eluted together, as are $(-)$-alkyl Lglycosides and $(+)$-alkyl D-glycosides. Therefore, the g.l.c. data for the various (-)-2-butyl L-glycosides can be obtained from the gas chromatograms of the corresponding (+)-2-butyl D-glycosides. In the Figures, to indicate the resolution of $\mathbf{D}$ and $L$ enantiomers of the various sugars, the peaks derived from the different anomers of the trimethylsilylated $(+)-2$-butyl D-glycosides have been assigned as L. Commer- 
cial (-)-2-butanol contains $\sim 6 \%$ of the $(+)$-enantiomer, as shown by the presence of small peaks marked * in the Figures. The $(+)$-2-butanol sample was contaminated with $\sim 7 \%$ of the (-)-enantiomer.

In Table I, the relative retention times $(T)$ of the trimethylsilylated (-)- and $(+)$-2-butyl D-glycosides are presented. In addition, the corresponding peak-area

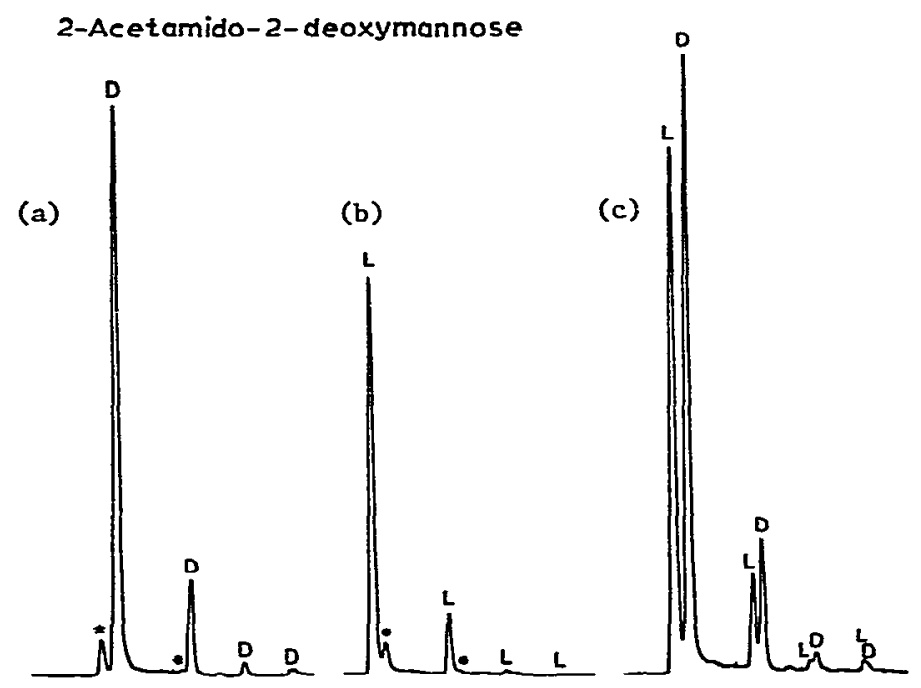

Fig. 1. G.1.c. pattern of trimethylsilylated derivatives of $(a)(-)-2-b u t y l$ 2-acetamido-2-deoxy-Dmannoside, $(b)(+)$-2-butyl 2-acetamido-2-deoxy-D-mannoside $[=(-)$-2-butyl 2-acetamido-2deoxy-L-mannoside; see text], and $(c)$ a mixture of both.

2-Acetamido-2-deoxygalactose

(a)

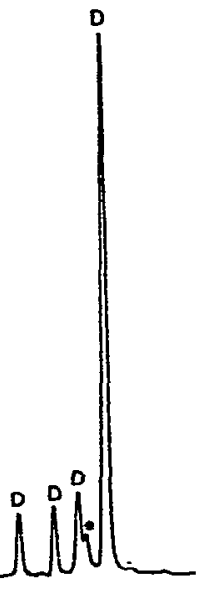

(b)

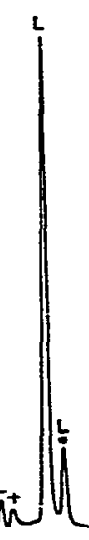

(c)

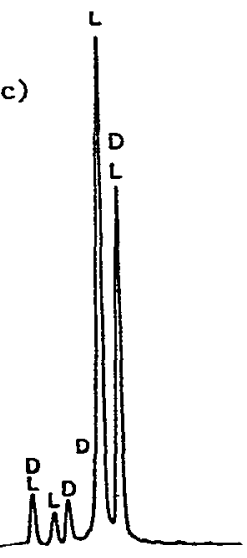

Fig. 2. G.l.c. pattern of trimethylsilylated derivatives of $(a)(-)$-2-butyl 2-acetamido-2-deoxy-Dgalactoside, (b) $(+)$-2-butyl 2-acetamido-2-deoxy-D-galactoside $[=(-)$-2-butyl 2-acetamido-2deoxy-L-galactoside; see text], and (c) a mixture of both. tUnknown product. 
2-Acetamido-2-deoxyglucose

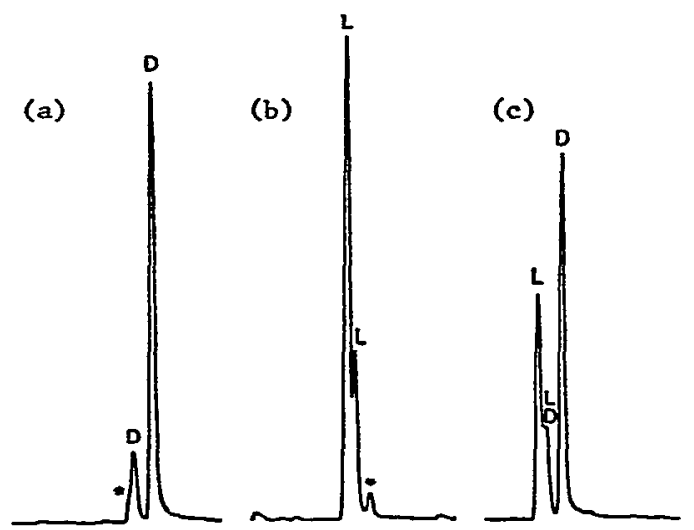

Fig. 3. G.l.c. pattern of trimethylsilylated derivatives of $(a)(-)$-2-butyl 2-acetamido-2-deoxy-Dglucoside, $(b)(+)$-2-butyl 2-acetamido-2-deoxy-D-glucoside $[=(-)$-2-butyl 2-acetamido-2-deoxy-Lglucoside; see text], and (c) a mixture of both.

Galacturonic acid

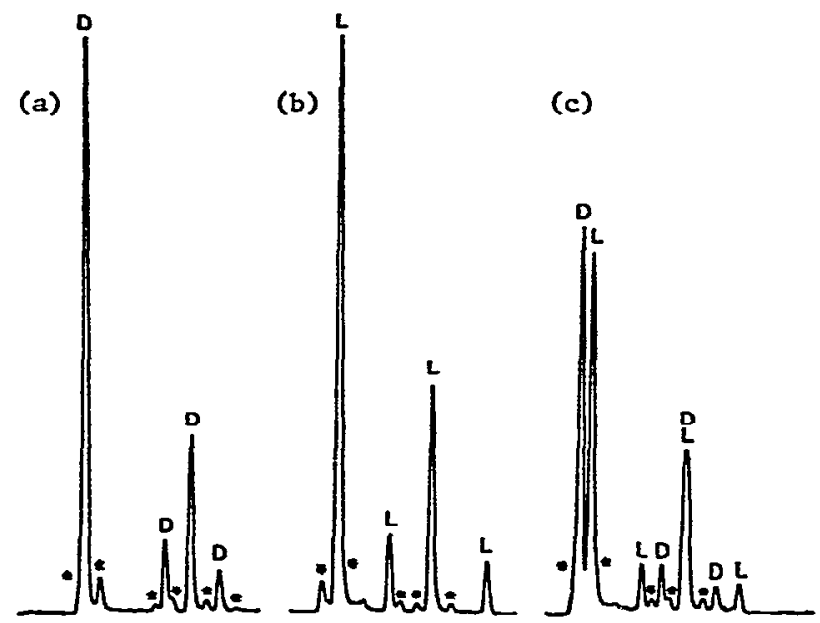

Fig. 4. G.l.c. pattern of trimethylsilylated derivatives of $(a)(-)$-2-butyl glycoside of D-galacturoniz acid $(-)$-2-butyl ester, $(b)(+)$-2-butyl glycoside of D-galacturonic acid $(+)$-2-butyl ester [ $=(-)$-2butyl glycoside of L-galacturonic acid (-)-2-butyl ester; see text], and (c) a mixture of both.

proportions and the mass-spectrometric data used for the assignment of the ring size are given ${ }^{1.3}$. The $\alpha$ and $\beta$ configurations of the different anomers were not determined. As found for the neutral monosaccharides, the absolute configurations of the 2-acetamido-2-deoxy sugars and uronic acids can be determined by capillary g.l.c. of the trimethylsilylated ( - )-2-butyl glycosides [or (+)-2-butyl glycosides]. All gas chromatograms showed good resolutions between $D$ and $L$ for most of the anomers. 


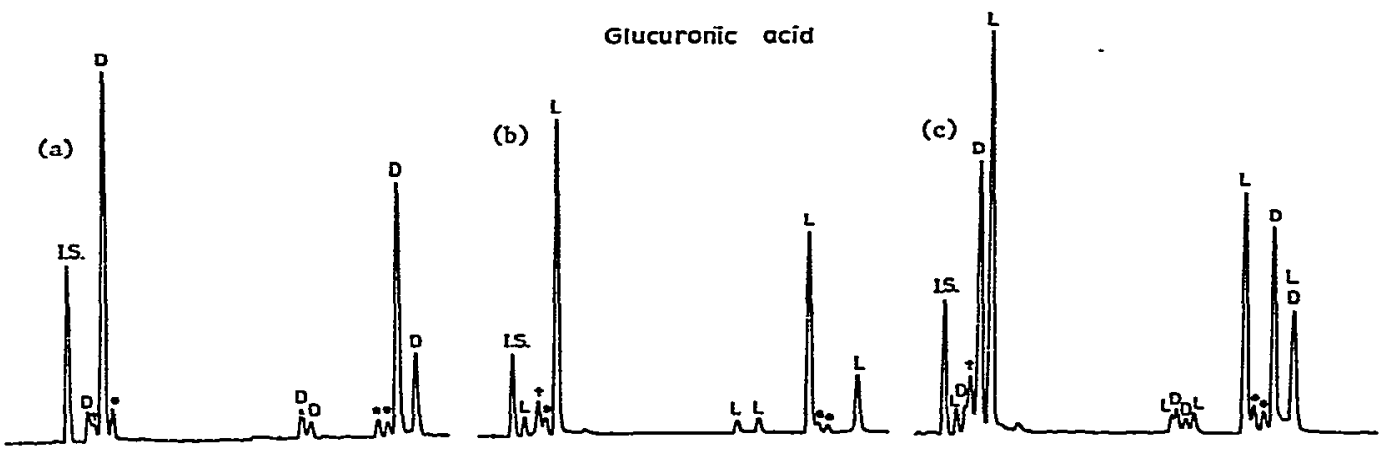

Fig. 5. G.I.c. pattern of trimethylsilylated derivatives of $(a)(-)$-2-butyl glycoside of D-glucuronic acid (-)-2-butyl ester and lactone, $(b)(+)$-2-butyl glycoside of D-glucuronic acid $(+)$-2-butyl ester and lactone $[=(-)$-2-butyl glycoside of L-glucuronic acid ( - -)-2-butyl ester and lactone; see text], and $(c)$ a mixture of both. UUnknown product.

During glycosidation of the 2-acetamido-2-deoxy sugars with 2-butanol, the $N$-acetyl group is partially cleaved (manno $\sim 95 \%$, galacto $\sim 65 \%$, gluco $\sim 30 \%$ ). A re- $N$-acetylation step is necessary and special conditions are necessary to minimise $O$-acetylation. Trimethylsilylated 2-butyl glycosides of 2-amino-2-deoxy sugars give peaks in the region $T 1.09-1.39$, where the neutral monosaccharide derivatives are also eluted. 2-Acetamido-2-deoxy-D-galactose is very sensitive to this $O$-acetylation (small peaks, $T 1.92$ and 1.98 ).

The glycosidation of uronic acids with ( \pm )-2-butanol yields ester glycosides, and four diastereomers are possible for each anomer. For the major furanoid form of D-galacturonic acid and the major pyranoid form of D-glucuronic acid, a complete resolution of the four diastereomers was obtained (D-galacturonic acid: $T$ 1.45, 1.46 $[-,-], 1.49[+,+]$, and 1.50; D-glucuronic acid: $T 1.71[+,+], 1.73,1.76$, and $1.78[-,-])$. Table I contains only the data for the acids treated with $(-)$ - and $(+)$-2-butanol. Glucuronic acid yields reproducible amounts of the 2-butyl glycosides of the $\alpha$ and $\beta$ 6,3-lactone, and peaks of the latter substances can be used also for the assignment of the absolute configuration (see Fig. 5).

The method was then applied to various types of biopolymer.

The capsular polysaccharide isolated from Klebsiella type 57 was methanolysed $^{3,4}$ and the resulting mixture of methyl glycosides was butanolysed with (-)-2butanol and trimethylsilylated. The gas chromatogram of the products indicated that all of the sugar compounds (mannose, galactose, and galacturonic acid) belong to the $D$ series, in accord with the literature data ${ }^{5}$.

$\alpha_{1}$-Acid glycoprotein, when subjected to methanolysis, re- $N$-acetylation, butanolysis, re- $N$-acetylation, and trimethylsilylation, gave the (-)-2-butyl glycosides of L-fucose, D-mannose, D-galactose, and 2-acetamido-2-deoxy-D-glucose. The D and $\mathrm{L}$ enantiomers of $N$-acetylneuraminic acid 2-butyl ester ( $T$ 2.40) cannot be separated under the conditions used. For galactose and 2-acetamido-2-deoxygalactose, present in mucus glycoproteins, the $\mathrm{D}$ configuration was confirmed.

The method was also applied to a highly complex, methylated, acidic poly- 


\section{TABLE I}

G.L.C. AND MASS-SPECTROMETRIC DATA FOR THE TRIMETHYLSILYLATED (-)-2-BUTYL GLYCOSIDES AND (+)-2-BUTYL GLYCOSIDES OF SOME 2-ACETAMIDO-2-DEOXY-D-HEXOSES AND D-HEXURONIC ACIDS. URONIC ACIDS ARE ANALYSED AS 2-BUTYL ESTERS

$A$, reaction with (-)-2-butanol; $B$, reaction with (+)-2-butanol. G.1.c. data for $(+)$-2-butyl derivatives of $D$ sugars are equal to those for (-)-2-butyl derivatives of $L$ sugars, and conversely. The relative retention-times are given with respect to trimethylsilylated methyl $\alpha$-D-galactopyranoside $(\sim 31 \mathrm{~min}$ at a temperature programme of $135 \rightarrow 220^{\circ}$ at $1 \% \mathrm{~min}$ ).

\begin{tabular}{|c|c|c|c|c|}
\hline Parent sugar & $\mathrm{T}^{a}$ & $\%^{b}$ & Mass spectrometry & Ring size \\
\hline \multirow{3}{*}{$\begin{array}{l}\text { 2-Acetamido-2-deoxy-D-mannose } \\
(A)\end{array}$} & $\begin{array}{l}1.53 \\
1.68\end{array}$ & $\begin{array}{l}79 \\
16\end{array}$ & $\begin{array}{l}m / e 173 \gg m / e 186 \\
m / e 173 \gg m / e 186\end{array}$ & $p$ \\
\hline & $\begin{array}{l}1.68 \\
1.79\end{array}$ & 3 & $\begin{array}{l}m / e 173 \gg m / e 186 \\
m / e 173<m / e 186 ; m / e 205\end{array}$ & $\begin{array}{l}p \\
f\end{array}$ \\
\hline & 1.89 & 2 & $m / e 173<m / e 186 ; m / e 205$ & $f$ \\
\hline \multirow[t]{4}{*}{$(B)$} & 1.50 & 82 & $m / e 173 \gg m / e 186$ & $\boldsymbol{p}$ \\
\hline & 1.66 & 15 & $m / e 173 \gg m / e 186$ & $p$ \\
\hline & 1.77 & 2 & $m / e 173<m / e 186 ; m / e 205$ & $f$ \\
\hline & 1.89 & & $m / e 173<m / e$ 186; m/e 205 & $f$ \\
\hline \multirow{4}{*}{$\begin{array}{l}\text { 2-Acetamido-2-deoxy-D-galactose } \\
\text { (A) }\end{array}$} & 1.68 & 8 & $m / e 173<m / e 186 ; m / e 205$ & $f$ \\
\hline & 1.76 & 8 & $m / e 173<m / e 186 ; m / e 205$ & $f$ \\
\hline & 1.81 & 10 & $m / e 173>m / e 186$ & $p$ \\
\hline & 1.86 & 74 & $m / e 173>m / e 186$ & $p$ \\
\hline \multirow[t]{4}{*}{ (B) } & 1.68 & 3 & $m / e 173<m / e 186 ; m / e 205$ & $f$ \\
\hline & 1.72 & 5 & $m / e 173<m / e 186 ; m / e 205$ & $f$ \\
\hline & 1.82 & 83 & $m / e 173>m / e 186$ & $p$ \\
\hline & 1.86 & 9 & $m / e 173>m / e 186$ & $p$ \\
\hline \multirow{4}{*}{$\begin{array}{l}\text { 2-Acetamido-2-deoxy-D-glucose } \\
\text { (A) } \\
\text { (B) }\end{array}$} & 1.90 & 18 & $m / e 173 \gg m / e 186$ & $p$ \\
\hline & 1.93 & 82 & $m / e 173 \gg m / e .186$ & $p$ \\
\hline & 1.89 & 77 & $m / e 173 \gg m / e 186$ & $p$ \\
\hline & 1.90 & 23 & $m / e 173 \gg m / e 186$ & $p$ \\
\hline \multirow{4}{*}{$\begin{array}{l}\text { D-Galacturonic acid } \\
\text { (A) }\end{array}$} & 1.46 & 68 & $m / e 204 \ll m / e 217$ & $f$ \\
\hline & 1.63 & 8 & $m / e 204 \ll m / e 217$ & $f$ \\
\hline & 1.68 & 20 & $m / e 204>m / e 217$ & $p$ \\
\hline & 1.74 & 4 & $m / e 204>m / e 217$ & $p$ \\
\hline \multirow[t]{4}{*}{ (B) } & 1.49 & 62 & $m / e 204 \ll m / e 217$ & $f$ \\
\hline & 1.59 & 8 & $m / e 204 \ll m / e 217$ & $f$ \\
\hline & 1.68 & 24 & $m / e 204>m / e 217$ & $p$ \\
\hline & 1.79 & 6 & $m / e 204>$ m/e 217 & $p$ \\
\hline \multirow{6}{*}{$\begin{array}{l}\text { D-Glucuronic acid } \\
\text { (A) }\end{array}$} & 1.05 & 3 & lactone & \\
\hline & 1.08 & 46 & lactone & \\
\hline & 1.55 & 4 & $m / e 204 \ll m / e 217$ & $\tilde{J}$ \\
\hline & 1.57 & 2 & $m / e 204 \ll m / e 217$ & $f$ \\
\hline & $\mathbf{1 . 7 8}$ & 34 & $m / e 204>m / e 217$ & $p$ \\
\hline & 1.83 & 11 & $m / e 204>m / e 217$ & $p$ \\
\hline \multirow[t]{6}{*}{ (B) } & 1.03 & 2 & lactone & \\
\hline & 1.11 & 46 & lactone & \\
\hline & 1.54 & 2 & $m / e 204 \ll m / e 217$ & $f$ \\
\hline & 1.59 & 3 & $m / e 204 \ll m / e 217$ & $f$ \\
\hline & 1.71 & 35 & $m / e 204>m / e 217$ & $p$ \\
\hline & 1.83 & 12 & $m / e 204>$ m/e 217 & $p$ \\
\hline
\end{tabular}

aIt was found that different SE-30 capillary columns give rise to small, systematic deviations in $T$ values. The values given in the Table and those published earlier ${ }^{1}$ were obtained on the same SE-30 column. ${ }^{\circ}$ Comparison of the relative intensities of the peaks obtained from corresponding (-)-2butyl and ( + )-2-butyl D-glycosides sometimes shows differences which cannot easily be explained as a result of standard deviation. 
saccharide isolated from the coccoliths of Emiliania huxleyi (Lohmann) Kamptner. L-Galactose, D-glucose, D-mannose, L-mannose, L-rhamnose, L-arabinose, D-ribose, D-xylose, 6- $O$-methyl-D-mannose, 6-O-methyl-L-mannose, 2,3-di- $O$-inethyl-L-rhamnose, 3- $O$-methyl-D-xylose, and D-galacturonic acid ${ }^{6}$ were identified as constituent sugars. This is the first example in which mannose (DL ratio, 9:1) and 6-O-methylmannose (DL ratio, 1:2) occur in both enantiomeric forms.

The foregoing method can be incorporated in quantitative, analytical methods for monosaccharides which use alditol acetates ${ }^{7}$ or methyl glycosides ${ }^{4}$. Using the procedure of Clamp et al. ${ }^{4}$, after methanolysis and re- $N$-acetylation, one part of the mixture of methyl glycosides can be analysed quantitatively as the trimethylsilyl derivatives; the other part can be butanolysed and further derivatised for determination of absolute configuration. The choice of the internal standard applied for the quantitative analysis depends on the monosaccharides present. Arabinitol ( $T 0.80)$ coincides partially with the second peak of (-)-2-butyl D-fucoside ( $T 0.79)$, mannitol $(T$ 1.33) with the first peak of $(-)$-2-butyl L-glucoside $(T$ 1.32), and meso-inositol $(T$ 1.72) with the fifth peak of (-)-2-butyl glycoside of L-gluclironic acid (-)-2-butyl ester ( $T$ 1.71).

EXPERIMENTAL

(-)-2-Butanol (purum), (+)-2-butanol (purum), and ( \pm )-2-butanol (purum) were obtained from Fluka AG. Alcoholic solutions of $M$ hydrochloric acid were prepared as described by Clamp et al. ${ }^{4}$, and stored at $-18^{\circ}$ in a desiccator. viously ${ }^{1}$.

Capillary g.l.c. and g.l.c.-mass spectrometry were performed as reported pre-

Derivatization procedure for 2-acetamido-2-deoxyhexoses. - Nitrogen was bubbled through a solution of dry monosaccharide $(0.5 \mathrm{mg})$ in 2-butanolic $\mathrm{M} \mathrm{HCl}$ $(0.5 \mathrm{ml})$ for $30 \mathrm{sec}$, and the ampoule was then sealed. After butanolysis for $8 \mathrm{~h}$ at $80^{\circ}$, the solution was neutralised with $\mathrm{Ag}_{2} \mathrm{CO}_{3}$. $\mathrm{Re}-\mathrm{N}$-acetylation was effected by the addition of 10-25 $\mu \mathrm{l}$ of acetic anhydride. This mixture was kept at room temperature for $4 \mathrm{~h}$ : The precipitate was triturated thoroughly and centrifuged ( $2000 \mathrm{~g}$ for $10 \mathrm{~min}$ ), and the supernatant solution was concentrated under reduced pressure at $45^{\circ}$. The residue was dried for $12 \mathrm{~h}$ in vacuo over $\mathrm{P}_{2} \mathrm{O}_{5}$, and then treated with hexamethyldisilazane-chlorotrimethylsilane-pyridine $(0.1 \mathrm{ml}, 1: 1: 5)$ for $30 \mathrm{~min}$ at room temperature.

Derivatization procedure for neutral monosaccharides and uronic acids. - The same procedure was followed as described above, except that the re- $N$-acetylation step was omitted ${ }^{1}$.

\section{ACKNOWLEDGMENTS}

We thank Mr. C. Versluis and Dr. H. W. J. Spronck (Laboratory of Analytical Chemistry, University of Utrecht) and Dr. Ir. P. A. Leclercq (Department of Mass 
Spectrometry, University of Eindhoven) for recording the mass spectra. This investigation was supported by the Netherlands Foundation for Chemical Research (SON) with financial aid from the Netherlands Organization for the Advancement of Pure Research (ZWO).

\section{REFERENCES}

1 G. J. GeRWig, J. P. Kamerling, ANd J. F. G. Vliegenthart, Carbohydr. Res., 62 (1978) 349-357.

2 K. LeONTEIN, B. LINDBERG, AND J. LöNNGREN, Carbohydr. Res., 62 (1978) 359-362.

3 J. P. Kamerling, G. J. GerWig, J. F. G. Vliegenthart, and J. R. Clamp, Biochem. J., 151 (1975) 491-495.

4 J. R. Clamp, T. BHATtI, AND R. E. Chambers, Methods Biochem. Anal., 19 (1971) 229-344.

5 J. P. Kamering, B. Lindberg, J. Lönngren, and W. Nimmich, Acta Chem. Scand., Ser. B, 29 (1975) 593-598.

6 A. M. J. Fichtinger-Schepman, J. P. Kamerling, J. F. G. Vliegenthart, E. W. de Jong, L. BosCH, AND P. Westbroex, Carbohydr. Res., 69 (1979) 181-189.

7 J. S. SAwardeker, J. H. Sloneker, AND A. R. Jeanes, Anal. Chem., 37 (1965) 1602-1604. 